\title{
ChemComm
}

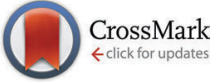

Cite this: Chem. Commun., 2015, 51, 12827

Received 7th May 2015

Accepted 8th July 2015

DOI: $10.1039 / \mathrm{c} 5 \mathrm{cc} 03817 \mathrm{k}$

www.rsc.org/chemcomm

\section{Photodimerisation of a coumarin-dipeptide gelator}

\author{
Emily R. Draper, Tom O. McDonald and Dave J. Adams*
}

\begin{abstract}
Here we report a coumarin based hydrogelator that can form bulk gels, or homogeneous thin gels via an electrochemical pH drop. The gel can then be strengthened by post-gelation photodimerisation of the coumarin groups by irradiating with UV light.
\end{abstract}

Low molecular weight gelators (LMWGs) self-assemble in a solvent to form fibres. At a sufficiently high concentration, the fibres entangle and cross-link to form a network, immobilising the solvent. ${ }^{1-3}$ The mechanical properties of the gel arise from the average thickness and mechanical properties of the fibres, the degree of branching (i.e. the distance between the cross-linking points), the type of cross-link (i.e. branching or entanglements) and how the fibres are distributed across the whole gel. ${ }^{4}$ These properties are difficult to control and there is limited information available for these systems. ${ }^{3,5}$

The self-assembly is driven by non-covalent interactions. This means that the gels are often reversible as (for example) heating is often sufficient to break these non-covalent bonds and so the LMWG re-dissolves. The cross-links between fibres are also such that the gels often break at relatively low strains as compared to polymeric gels. As a result, stretching for example is often impossible. For some applications, these properties can be desirable, but for others they can be a drawback. Hence, it is of interest to be able to covalently cross-link or post-modify a gel once formed to lock in the structure.

A number of methods have been used to post-modify LMWGs after gel formation. For example, tyrosine-containing gelators can be cross-linked by the formation of dityrosine, which has been shown to enhance the mechanical properties of the gels. ${ }^{6}$ An increase in the rheological properties of gels has also been reported by the reaction of an amine-containing LMWG with glutaraldehyde post-gel formation. ${ }^{7}$ Click chemistry has also been used to cross-link LMWG fibres post gelation,

Department of Chemistry, University of Liverpool, Crown Street, Liverpool, L69 7ZD, UK. E-mail: d.j.adams@liverpool.ac.uk

$\dagger$ Electronic supplementary information (ESI) available. See DOI: 10.1039/c5cc03817k and this cross-linking was shown to affect the rate of release of a drug entrapped in the network. ${ }^{8}$

There are a number of photoreactive moieties, including conjugated diyne units, ${ }^{9,10}$ alkynes, and diazides, ${ }^{11}$ which have been used to strengthen materials post-gelation. There are also many examples of photoisomerising groups such as stilbene ${ }^{12}$ and azobenzene. ${ }^{13-15}$ These often lead to a conformational change of the molecules from the gelling trans-isomer to the non-gelling cis-isomer or vice versa. This post-gelation modification is useful for surface photopatterning applications. ${ }^{16}$ Anthracene and coumarins are known to photodimerise when irradiated with light $>280 \mathrm{~nm} \cdot{ }^{17,18}$ The dimerisation of coumarins is reversible when light with a wavelength of less than $260 \mathrm{~nm}$ is used. The $[2+2]$ photodimerisation of coumarins is known to occur both in solution and in the solid state, often to give a mixture of syn-syn, syn-anti, anti-syn and anti-anti cyclobutanes. ${ }^{18,19}$

We hypothesised that incorporating coumarin into a LMWG would allow dimerisation within a self-assembled fibre to stiffen the fibre rather than reaction between fibres, as the coumarin molecules within neighbouring fibrils and fibres are unlikely to be close enough for dimerisation to happen. Either ought to lead to a change in the rheological properties of the fibres and hence a controllable change in the mechanical bulk properties of the gel, without destruction of the fibrous network. We note that Kim et al. have recently shown that by incorporating two coumarin groups into a $\beta$-sheet forming LMWG that the gel properties can be modified post-gelation with the use of UV light. ${ }^{20}$ This change in properties of the gel arises from the dimerisation of both coumarin units, which ultimately results in destruction of the gel due to disruption of fibres and formation of an insoluble network. Feng and coworkers have also incorporated a coumarin group into a LMWG to be used in cell imaging. ${ }^{21}$

The LMWG (Fig. 1a) is related to the many other dipeptidebased hydrogelators, where a large aromatic group is attached to the N-terminus. ${ }^{22}$ The gelator was prepared via the reaction of 7-hydroxycoumarin with tert-butyl chloroacetate, followed by removal of the tert-butyl group using acid catalysis. This carboxylic 

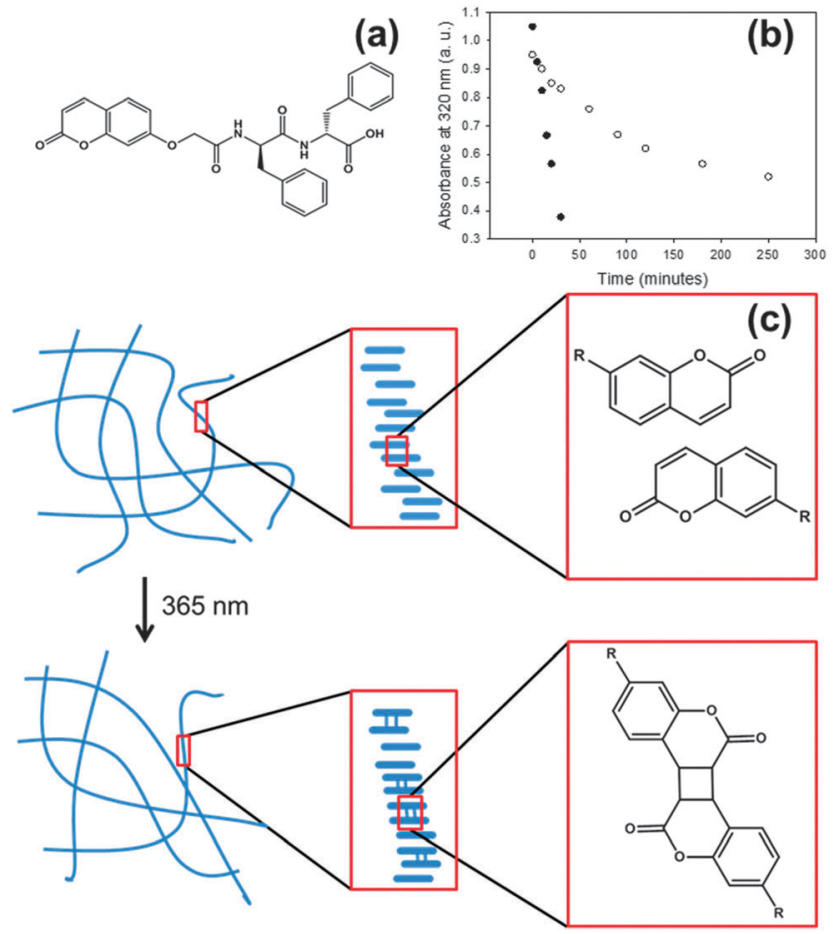

Fig. 1 (a) Molecular structure of the gelator. (b) Decrease in UV-vis absorption at $320 \mathrm{~nm}$ showing dimerization of gelator in a high $\mathrm{pH}$ solution (open circles) and in a gel at low pH (full circles). (c) Schematic showing possible stacking of gelator molecules in the gel before and after irradiation with UV light. Only one isomer of the coumarin dimer has been shown for clarity.

acid was then coupled to the $C$-ethyl protected diphenylalanine, followed by deprotection using lithium hydroxide to give the LMWG.

Gels were prepared by dissolving the gelator at high $\mathrm{pH}$ (typically $>10$ ), followed by reducing the $\mathrm{pH}$ by the addition of glucono- $\delta$-lactone (GdL). GdL hydrolyses slowly over time to gluconic acid, ${ }^{23}$ resulting in a homogeneous drop in $\mathrm{pH}$ as we have shown previously for related gelators. ${ }^{24}$ Using this approach, transparent, self-supporting gels are formed at a gelator concentration of $5 \mathrm{mg} \mathrm{mL} \mathrm{m}^{-1}$ (Fig. 2a). The properties of this gel are entirely consistent with gels of this type. The storage modulus $\left(G^{\prime}\right.$, $82000 \mathrm{~Pa}$ ) is approximately an order of magnitude higher than the loss modulus $\left(G^{\prime \prime}, 10000 \mathrm{~Pa}\right)$. The gels break at relatively low strain (1\%; Fig. S1, ESI $\dagger$ ). Scanning electron microscopy (SEM) shows that the gel arises from a network of fibres (Fig. 2b) with an average diameter of $42 \mathrm{~nm}$ (Fig. S2a, ESI $\dagger$ ). Fluorescence measurements show that the coumarin molecules form $\mathrm{H}$-aggregates upon gelation due to a blue shift in the emission spectra (Fig. S3, ESI†). FTIR for both GdL and electrochemically formed gels show peaks in the amide I region at 1620 and $1650 \mathrm{~cm}^{-1}$ which can be characteristic for anti-parallel $\beta$-sheets and random coil secondary structures respectively (Fig. S12, ESI $\dagger$ ). ${ }^{25}$ However, interpreting this data for short dipeptide molecules is often difficult as has been seen with other LMWGs. ${ }^{26}$

Irradiating the solution at high pH using a $365 \mathrm{~nm}$ LED shows that the absorbance at $320 \mathrm{~nm}$ readily reduces in intensity, consistent with photodimerisation ${ }^{27}$ (Fig. $1 \mathrm{~b}$ and Fig. S4, ESI†).

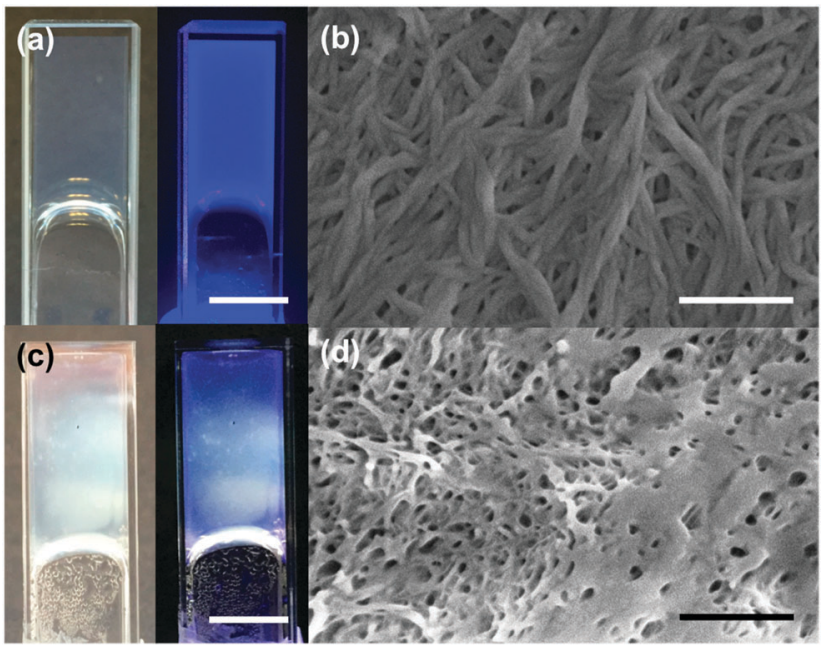

Fig. 2 Gels formed using GdL before irradiation (a) photographed under natural light (left) and under UV light (right), scale bar represents $1 \mathrm{~cm}$. (b) SEM image of GdL gel, scale bar represents $500 \mathrm{~nm}$. Gels formed by GdL after irradiation for 2 hours (c) photographed under natural light (left) and under UV light (right), scale bar represents $1 \mathrm{~cm}$. (d) SEM image after irradiation, scale bar represents $500 \mathrm{~nm}$.

In the gel state, the photodimerisation is significantly faster. We hypothesise that this is due to the coumarin molecules being closer to each other when self-assembled into fibres as compared to dispersed at high pH (Fig. 1b and Fig. S3, ESI $\dagger$ ). Photodimersation was further confirmed by mass spectrometry of the irradiated samples (Fig. S6, ESI $\dagger$ ). The degree of photodimerisation relates to the time over which the gel is exposed to UV light. The gel becomes slightly turbid on being exposed to UV light, with the turbidity being most pronounced at the front of the gel which is directly exposed to the LED (Fig. 2c and Fig. S7, ESI $\dagger$ ). This lack of homogeneity presents difficulties for analysing the effect of the irradiation on the rheological properties of the gels. This inhomogeneous change in gel network is also shown in the SEM images of the irradiated sample shown in Fig. $2 \mathrm{~d}$ with averages fibre diameters of $39 \mathrm{~nm}$ (Fig. S2b, ESI $\dagger$ ).

Hence, we prepared thin hydrogel films (of the order of $2 \mathrm{~mm}$ thick) utilising an electrochemical approach, which we have previously reported. Briefly, hydroquinone is electrochemically oxidised to provide a $\mathrm{pH}$ gradient at the electrode. ${ }^{28-30}$ This results in a gel being formed only at the electrode as opposed to in the bulk as shown in Fig. 3a, with the thickness of the gel being controlled by both the current used and the time for which the current is applied.

Using this approach, films could be formed on ITO-coated glass slides. The gels were carefully removed from the slide and the rheological properties measured. The absolute gel properties were weaker than those formed in the bulk, but were consistent in behaviour, with the gels breaking at relatively low strain and $G^{\prime}$ being independent of frequency (Fig. S8, ESI $\dagger$ ). This method of gelation was also found to give gels with very reproducible rheological properties (Fig. S9, ESI $\dagger$ ). To probe the effect of irradiation on the rheological properties, the gels were removed from the bulk liquid and sealed in a hydrated chamber. The gel 

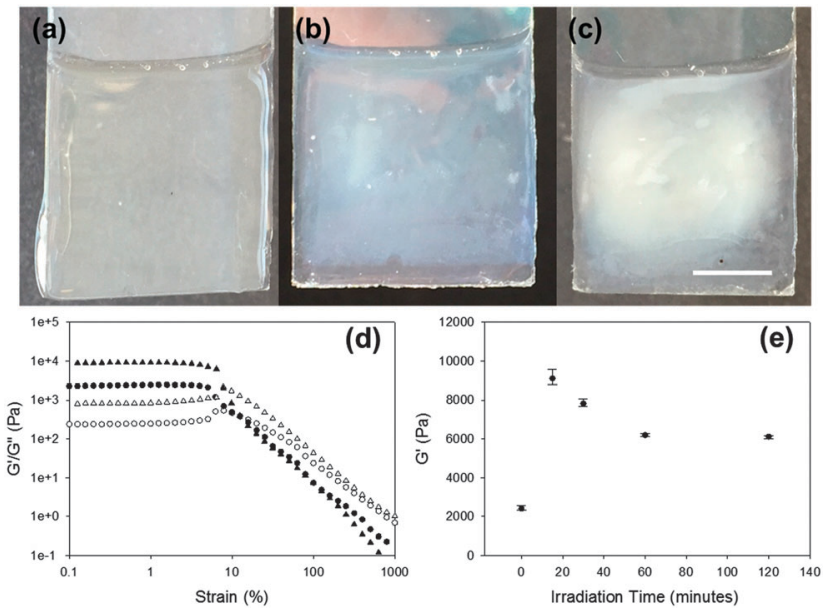

Fig. 3 Photographs of gels grown electrochemically (a) before irradiation, (b) after 15 minutes of irradiation with $365 \mathrm{~nm}$ LED and (c) after irradiation for 60 minutes, scale bar represents $1 \mathrm{~cm}$. (d) Rheological strain sweep data for electrochemically grown gels before irradiation (circles) and after 15 minutes of irradiation with a $365 \mathrm{~nm}$ LED (triangles). $G^{\prime}$ (open shapes) and $G^{\prime \prime}$ (full shapes). Measurements recorded at $10 \mathrm{rad} \mathrm{s}^{-1}$. (e) Graph showing the change in $G^{\prime}$ of electrochemically grown gels after increasing time under $365 \mathrm{~nm}$. Error bars are calculated from an average of four repeat measurements.

was then irradiated by a $365 \mathrm{~nm}$ LED through a small hole in the top of the chamber. The gel was moved periodically to ensure uniform exposure of the entirety of the sample to the LED. As the sample was irradiated, it became slightly more turbid (Fig. 3b). After one hour of irradiation the gel was more opaque (Fig. 3c). Using this thin film method, irradiation appeared more uniform over the entire gel, this can be seen clearly when the gels are viewed under UV light (Fig. S10, ESI $\dagger$ ). This was also seen when different parts of the gel were dissolved in DMSO and the UV-vis spectra recorded showing similar amounts of dimer present (Fig. S11, ESI $\dagger$ ).

Measuring the rheological properties of the gels shows that that after 15 minutes of irradiation with a $365 \mathrm{~nm}$ LED, $G^{\prime}$ and $G^{\prime \prime}$ both increased compared to the original gel (Fig. 3d). Further irradiation time resulted in a decrease in the rheological properties compared to that after 15 minutes, but the gels were still stiffer than before irradiation. Similar effects have recently been reported by Kim et $a .^{20}$ After 60 minutes irradiation, there was no further decrease in $G^{\prime}$, but the turbidity of the gel noticeably increased (Fig. 3e).

We attribute the increase in the rheological properties after 15 minutes to the photodimerisation of the coumarin group and dimerisation of coumarin gelator molecules. This dimerisation strengthens the fibres in the network due to the formation of covalent bonds between molecules rather than just non-covalent. Since we have only one coumarin group per gelator, complete cross-linking of the fibres is not possible. We rather envisage that this photodimerisation leads to an increase in stiffness of the individual fibres. That further irradiation resulted in a decrease $G^{\prime}$ is an interesting observation. We attribute this to the fibres stiffening to a sufficient degree that the dimerized network is disrupted. When comparing the IR of the irradiated electrochemically formed gels with the gels formed by GdL, both gels are very similar. Therefore, we conclude that if any hydroquinone radicals have been formed by UV light, these are not interacting or reacting with the fibres to affect their properties (Fig. S12, ESI $\dagger$ ). As a control, we ruled out drying effects by placing a gel in the same chamber used for the irradiation; after 2 hours, the appearance and rheological properties of the gel were not changed compared to a fresh gel (Fig. S13, ESI $\dagger$ ). The temperature of the gel during irradiation was also monitored. A $2{ }^{\circ} \mathrm{C}$ rise was measured, and hence it is unlikely that the changes in the rheological properties are due to an increase in temperature. We ruled out the dimer being able to self-assemble and increasing the $G^{\prime}$ of the gel by forming the dimer in solution by irradiation for two hours. The $\mathrm{pH}$ was then lowered using GdL. Instead of gelation occurring, a precipitate was formed (Fig. S14, ESI $\dagger$ ). The dimer does not form a gel and so is unlikely to be selfassembling and strengthening the gel (for example, as a second independent, self-sorted network). ${ }^{31}$ An irradiated gel formed by GdL was also re-dissolved using a small amount of sodium hydroxide solution. The same was done for a gel that had not been irradiated. The $\mathrm{pH}$ of both these solutions was lowered again using GdL. The irradiated gel did not gel but the nonirradiated gel did (Fig. S15, ESI†). This again demonstrates that the dimer does not gel.

Before irradiation, SEM images of electrochemically formed gels show a random fibrous network (Fig. S17a, ESI $\dagger$ ), similar to that seen for gels formed by the GdL method. After an hour of irradiation, SEM images show that the network has changed, instead showing less defined structure, again as for gels formed with GdL (Fig. S17b, ESI $\dagger$ ). Electrochemically grown gels show a more uniform change in the structure as also seen in the change in transparency of the gels. Both gels show significant change in the morphology of the gel with smooth featureless areas that could be due to surface effects.

The effect of light intensity was also investigated. The more intense the light, the greater the increase in $G^{\prime}$ after 15 minutes of irradiation (Fig. S18, ESI $\dagger$ ). This is due to the dimerisation being slower due to the lower light intensity. As the dimerisation of the coumarin molecule is reversible with wavelengths $<260 \mathrm{~nm}$, the irradiated samples were then irradiated with $254 \mathrm{~nm}$ light for 5 hours. However, there was no change in the samples visibly or by NMR (Fig. S16, ESI $\dagger$ ) but we acknowledge this could be due to the power output of the light source used.

In summary, we have synthesised a coumarin-based dipeptide gelator that is able to form hydrogels using a pH switch, using both GdL and hydroquinone. Both gels are able to photodimerise after irradiation with a $365 \mathrm{~nm}$ LED. This irradiation leads to an increase in the rheological properties, this is believed to be caused by the dimerisation within fibres stiffening them, rather than cross-linking being formed between fibres. This opens up the possibilities to enhance gels post-gelation, or the locking in a structure by the covalent dimerisation. The use of UV light could also be used to photopattern surfaces for applications such as cell culture and differentiation.

ED thanks the EPSRC for a DTA studentship. DA thanks the EPSRC for a Fellowship (EP/L021978/1). 


\section{Notes and references}

1 P. Terech and R. G. Weiss, Chem. Rev., 1997, 97, 3133-3160.

2 S. Datta and S. Bhattacharya, Chem. Soc. Rev., 2015, DOI: 10.1039/ C5CS00093A.

3 J. Raeburn, A. Zamith Cardoso and D. J. Adams, Chem. Soc. Rev., 2013, 42, 5143-5156.

4 R. G. Weiss, J. Am. Chem. Soc., 2014, 136, 7519-7530.

5 J. Raeburn, C. Mendoza-Cuenca, B. N. Cattoz, M. A. Little, A. E. Terry, A. Zamith Cardoso, P. C. Griffiths and D. J. Adams, Soft Matter, 2015, 11, 927-935.

6 Y. Ding, Y. Li, M. Qin, Y. Cao and W. Wang, Langmuir, 2013, 29, 13299-13306.

7 M. A. Khalily, M. Goktas and M. O. Guler, Org. Biomol. Chem., 2015, 13, 1983-1987.

8 D. D. Diaz, E. Morin, E. M. Schon, G. Budin, A. Wagner and J.-S. Remy, J. Mater. Chem., 2011, 21, 641-644.

9 M. George and R. G. Weiss, Chem. Mater., 2003, 15, 2879-2888.

10 C. Kim, S. J. Lee, I. H. Lee, K. T. Kim, H. H. Song and H.-J. Jeon, Chem. Mater., 2003, 15, 3638-3642.

11 D. D. Díaz, J. J. Cid, P. Vázquez and T. Torres, Chem. - Eur. J., 2008, 14, 9261-9273.

12 S. Miljanić, L. Frkanec, Z. Meić and M. Žinić, Eur. J. Org. Chem., 2006, 1323-1334.

13 Y. Huang, Z. Qiu, Y. Xu, J. Shi, H. Lin and Y. Zhang, Org. Biomol. Chem., 2011, 9, 2149-2155.

14 C.-S. Chen, X.-D. Xu, S.-Y. Li, R.-X. Zhuo and X.-Z. Zhang, Nanoscale, 2013, 5, 6270-6274.
15 T. M. Doran, D. M. Ryan and B. L. Nilsson, Polym. Chem., 2014, 5, 241-248.

16 S. Khetan and J. A. Burdick, Soft Matter, 2011, 7, 830-838.

17 Y. Sako and Y. Takaguchi, Org. Biomol. Chem., 2008, 6, 3843-3847.

18 K. Tanaka, Molecules, 2012, 17, 1408-1418.

19 K. Gnanaguru, N. Ramasubbu, K. Venkatesan and V. Ramamurthy, J. Org. Chem., 1985, 50, 2337-2346.

20 S. H. Kim, Y. Sun, J. A. Kaplan, M. W. Grinstaff and J. R. Parquette, New J. Chem., 2015, 39, 3225-3228.

21 W. Ji, G. Liu, M. Xu, X. Dou and C. Feng, Chem. Commun., 2014, 50, 15545-15548.

22 S. Fleming and R. V. Ulijn, Chem. Soc. Rev., 2014, 43, 8150-8177.

23 Y. P. A. E. Green, J. Am. Chem. Soc., 1973, 95, 113-119.

24 D. J. Adams, M. F. Butler, W. J. Frith, M. Kirkland, L. Mullen and P. Sanderson, Soft Matter, 2009, 5, 1856-1862.

25 J. T. Pelton and L. R. McLean, Anal. Biochem., 2000, 277, 167-176.

26 M. G. Wolf, J. A. Jongejan, J. D. Laman and S. W. de Leeuw, J. Phys. Chem. B, 2008, 112, 13493-13498.

27 K. Muthuramu and V. R. Murthy, J. Org. Chem., 1982, 47, 3976-3979.

28 E. K. Johnson, L. Chen, P. S. Kubiak, S. F. McDonald, D. J. Adams and P. J. Cameron, Chem. Commun., 2013, 49, 8698-8700.

29 J. Raeburn, B. Alston, J. Kroeger, T. O. McDonald, J. R. Howse, P. J. Cameron and D. J. Adams, Mater. Horiz., 2014, 1, 241-246.

30 E. K. Johnson, D. J. Adams and P. J. Cameron, J. Am. Chem. Soc., 2010, 132, 5130-5136.

31 J. Raeburn and D. J. Adams, Chem. Commun., 2015, 51, $5170-5180$ 